\title{
Simulation of Gaussian electron guns for RHIC electron lens
}

\author{
A. Pikin
}

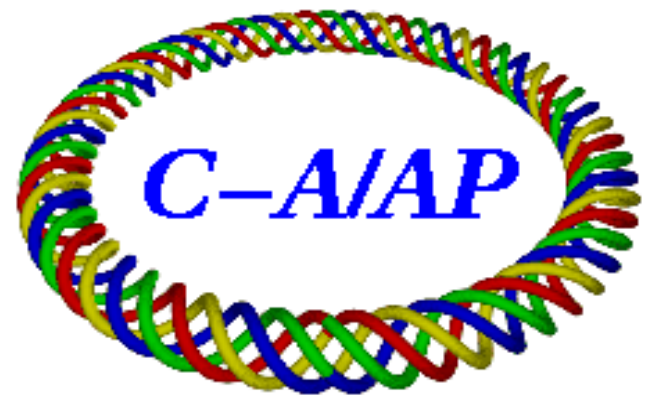

\section{Collider-Accelerator Department Brookhaven National Laboratory Upton, NY 11973}

Notice: This document has been authorized by employees of Brookhaven Science Associates, LLC under Contract No. DE-AC02-98CH10886 with the U.S. Department of Energy. The United States Government retains a nonexclusive, paid-up, irrevocable, world-wide license to publish or reproduce the published form of this document, or allow others to do so, for United States Government purposes. 


\title{
Simulation of Gaussian electron guns for RHIC electron lens
}

\author{
A. Pikin
}

February 28, 2014

\begin{abstract}
Simulations of two versions of the electron gun for RHIC electron lens are presented. The electron guns have to generate an electron beam with Gaussian radial profile of the electron beam density. To achieve the Gaussian electron emission profile on the cathode we used a combination of the gun electrodes and shaping of the cathode surface. Dependence of electron gun performance parameters on the geometry of electrodes and the margins for electrodes positioning are presented.
\end{abstract}

\section{First electron gun for electron lens $\left(d_{\text {cath }}=8.2 \mathrm{~mm}\right)$.}

The objective in simulation was to create an electron gun with Gaussian distribution of the emission current density, which is capable of generating the electron beams with currents up to $1 \mathrm{~A}$. The choice of the cathode diameter $\mathrm{d}_{\text {cath }}=8.2 \mathrm{~mm}$ was based on a required range of the beam Sigma in the interaction region $\sigma_{b}=0.28-0.78 \mathrm{~mm}$ for the magnetic field on the cathode $\mathrm{B}_{\text {cath }}=0.2-0.8 \mathrm{~T}$ and magnetic field in the interaction region $B_{\text {inter_region }}=2.5-6.0 \mathrm{~T}$ (for a magnetically confined beam). The required ratio of the beam radius to the beam Sigma was $r_{b} / \sigma_{b} \approx 3$.

Basically the structure of the electron gun electrodes is close to the Gaussian gun, built in Fermi Lab [1]. Our first several attempts to generate such cathode emission profile, which has small deviations from Gaussian distribution, using electric field forming by the gun electrodes and simple shapes of the cathode surface were not successful. Eventually we had to modify the initial spherical cathode surface to get the required emission profile. The general design of this electron gun along with other parts of the e-lens one can find in [2].

The RHIC e-lens electron gun has a control electrode positioned in a vicinity of the cathode similar to a Fermi Lab gun [2]. The simulation model with main dimensions is presented in Fig. 1

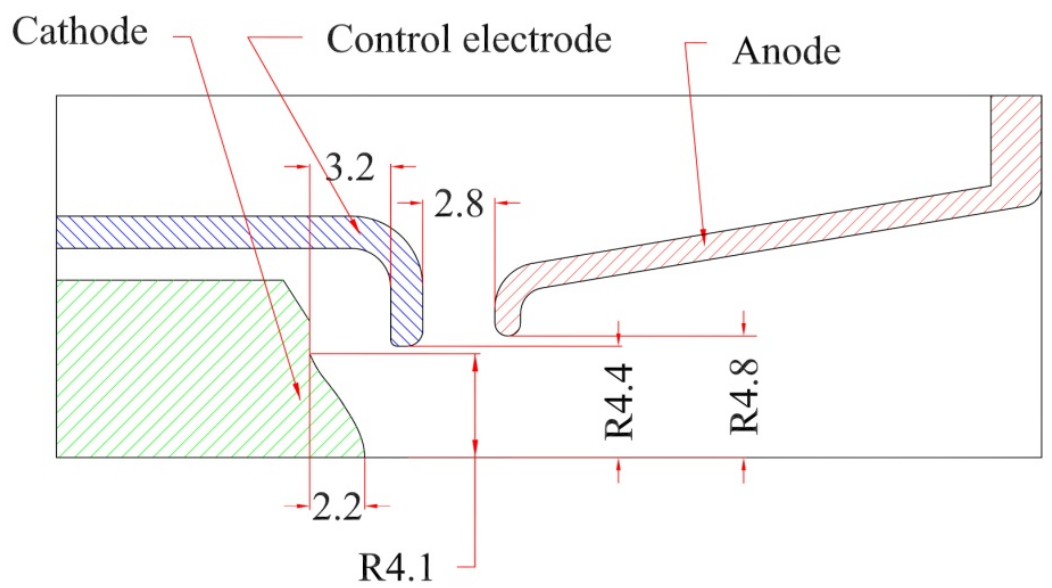

Fig. 1. Simulation model for a Gaussian electron gun with cathode diameter $8.2 \mathrm{~mm}$. The dimensions are in $\mathrm{mm}$. 
For a fixed geometry of the control electrode and the anode the shape of the cathode has been tuned in multiple iterations to achieve a Gaussian profile of the emission current density. The quality criterion of such shaping was an error parameter, defined as:

$$
E r=\frac{\sum_{1}^{n}\left(i_{\text {Gauss }}-i_{\text {sim }}\right)^{2}}{n \cdot A}
$$

Er - error parameter

$\mathrm{i}_{\text {Gauss }}$ - Gaussian fit of emission current density for a particular radius,

$i_{\text {sim }}$ - Simulated value of the emission current density for the same radius,

$\mathrm{n}$ - number of trajectories,

A - Amplitude of the fitted Gaussian profile.

Considering possibility of controlling the constant current density component (pedestal in radial current density distribution), which is independent on radius, with potential of the control electrode, a constant shift $\beta$ has been added to a fitting Gaussian function:

$$
i_{\text {Gauss }}=A \cdot \exp \left(-\frac{r^{2}}{2 \cdot \sigma^{2}}\right)+\beta
$$

All simulations has been done using 2-dimensional commercial TRAK package [3]. A basic simulated radial profile of the first electron gun for initial electrode geometry is presented in Fig. 2.

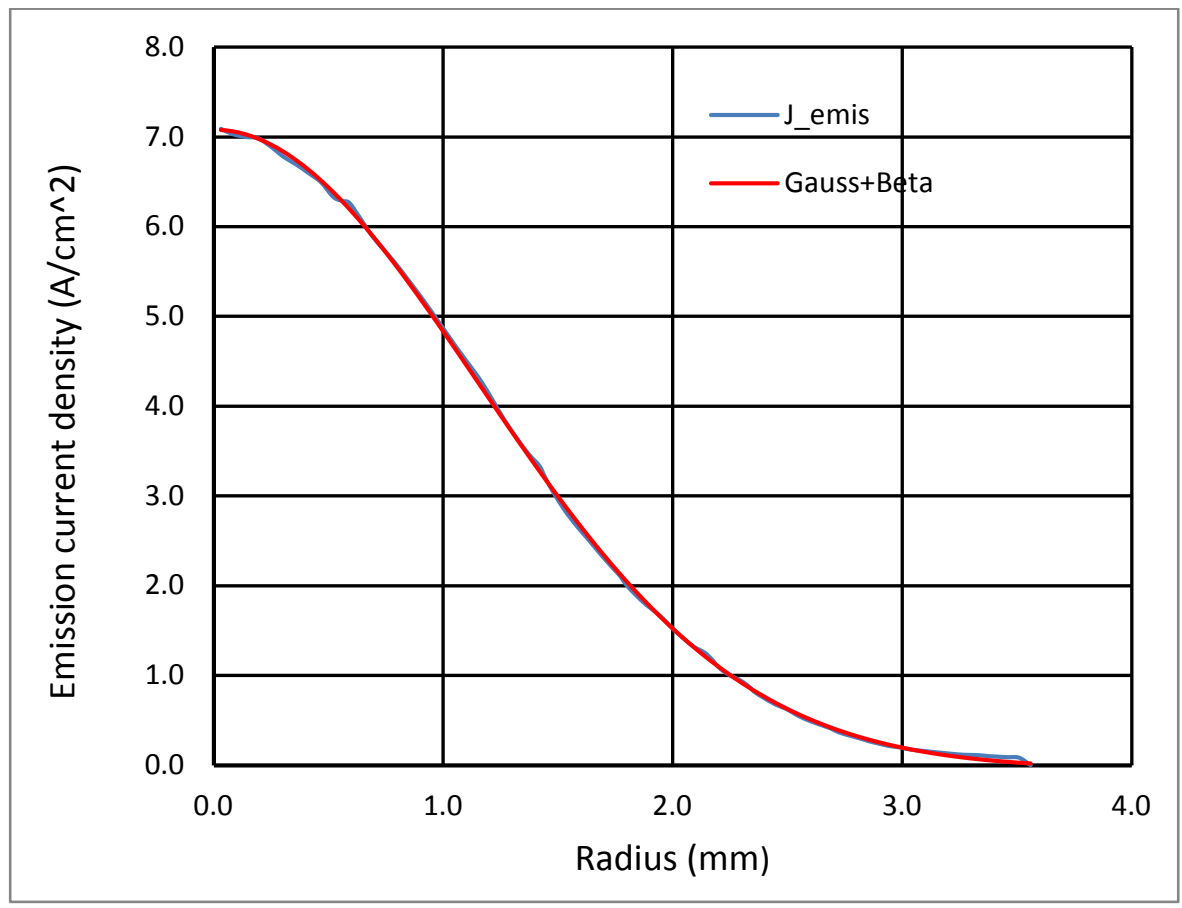

Fig. 2. Simulated radial profile of the emission current density of the first electron gun for anode voltage $U_{\mathrm{an}}=6.3 \mathrm{kV}$, control electrode voltage $\mathrm{U}_{\mathrm{ce}}=0.0 \mathrm{~V}$, electron current $\mathrm{I}_{\mathrm{el}}=0.59 \mathrm{~A}$. A fitting function has $A=7.12 \mathrm{~A} / \mathrm{cm}^{2}, \sigma=1.15 \mathrm{~mm}$ and $\beta=-0.038 \mathrm{~A} / \mathrm{cm}^{2}$. The value of Error parameter is $\mathrm{Er}=1.24 \mathrm{E}-4$. 
The electron beam current density distribution for this electron gun has been measure experimentally using a scintillator screen and a pinhole detector at the entrance into the electron collector and the results [4] confirmed these simulations. An option to control pedestal by changing the potential of the control electrode is demonstrated in Fig. 3.

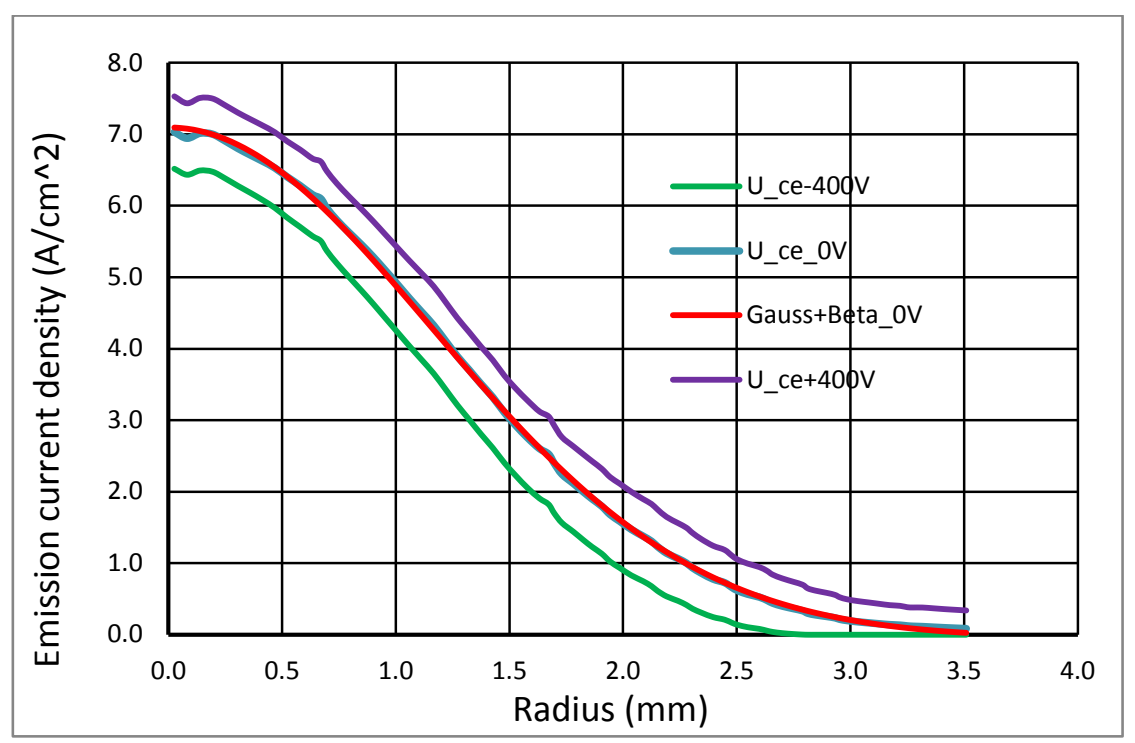

Fig. 3. Simulated radial profiles of the emission current density for different voltages on the control electrode for anode voltage $6.1 \mathrm{kV}$.

One can see that the voltage on the control electrode with respect to the cathode can visibly modify the emission profile, affecting both beam width (Sigma) and the total electron current. A condensed dependence of the pedestal $\beta$ on the control electrode voltage is presented in Fig. 4.

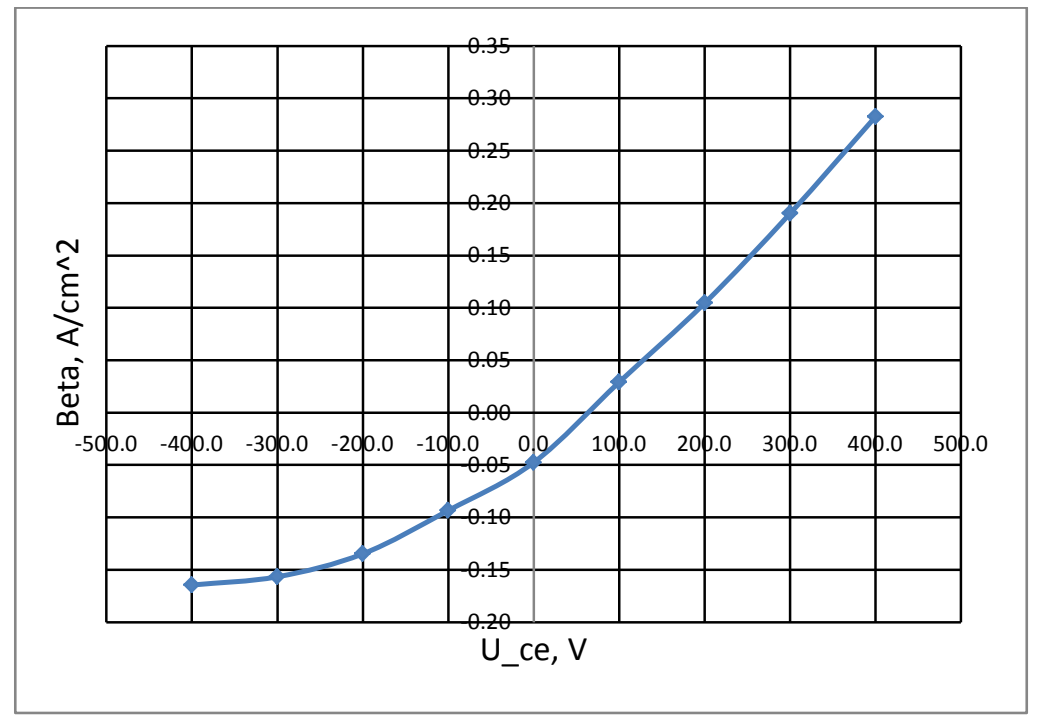


Fig. 4. Dependence of pedestal $\beta$ in a fitting function on the voltage on the control electrode with respect to the cathode.

With anode voltage $U_{a n}=10.0 \mathrm{kV}$ and control electrode voltage $U_{\mathrm{ce}}=0.0 \mathrm{~V}$ the maximum simulated electric field on the control electrode is $8.0 \mathrm{kV} / \mathrm{mm}$. The Kilpatrick safety factor for this voltage is 2.5 .

\section{Second electron gun (cathode diameter $d_{c a t h}=15.0 \mathrm{~mm}$ )}

A need for a larger cathode was dictated by the proton stability considerations in an interaction region primarily at "low" proton energy - around $100 \mathrm{GeV}$. Taking into account the existing limitations in the magnetic fields values on the cathode and in the interaction region, we decided to use a larger cathode diameter: $15.0 \mathrm{~mm}$. Apart from increasing the cathode diameter we also decided to increase a perveance of this gun to be able to get electron beam current more than $2 \mathrm{~A}$ with the available $10 \mathrm{kV}$ maximum voltage of the anode modulator and without compromising the Gaussian profile of the beam current density. Considering our previous experience with the first gun, we decided not to use isolated control electrode. Instead, it is permanently connected to the cathode. A requirement for the ratio of beam radius to a beam Sigma: $r / \sigma \approx 3$. It stays the same as for the first electron gun. Geometry of this gun is given in Fig. 5.

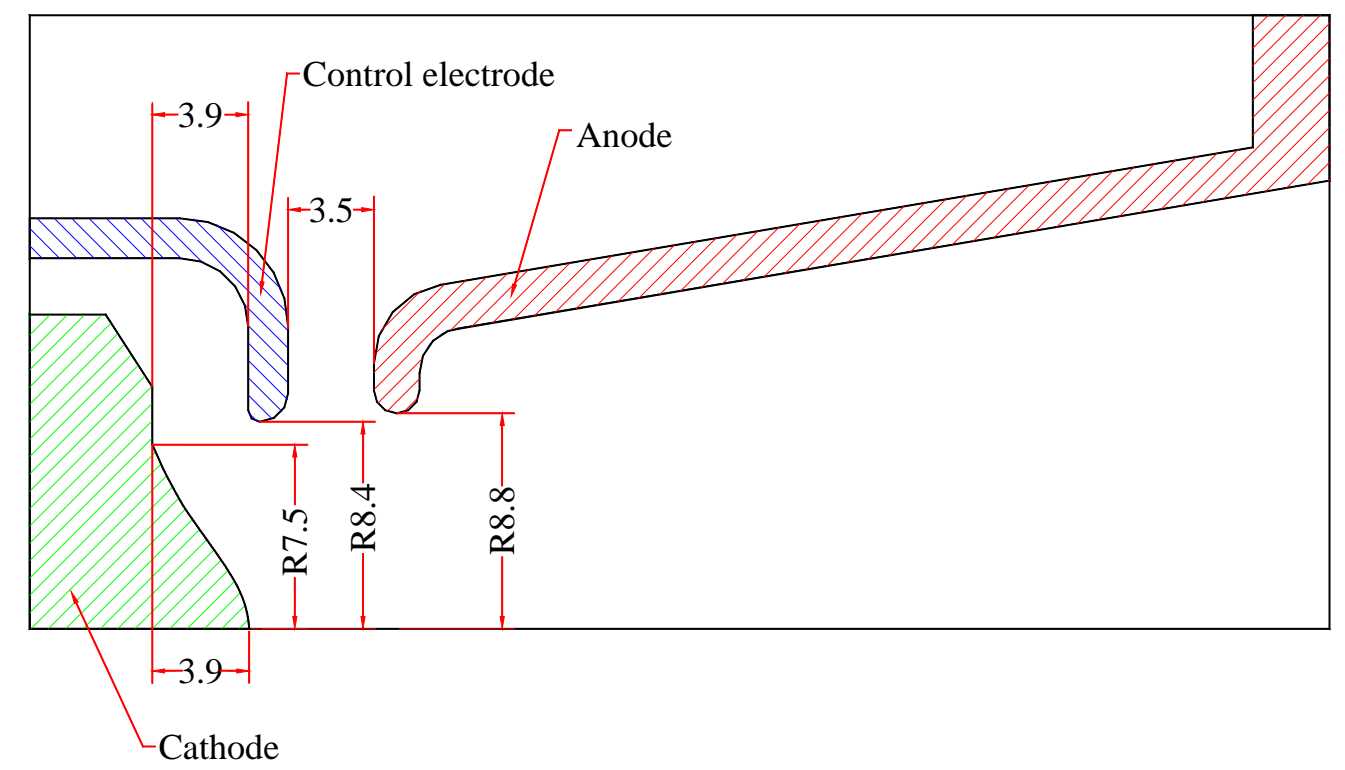

Fig. 5. Simulation model of the second electron gun with cathode diameter $15.0 \mathrm{~mm}$.

Considering requirement to increase the perveance of the gun, a new cathode shape has been generated for positions of the electrodes different from scaling of the first gun.

After tuning of the cathode surface for the geometry of electrodes presented in Fig. 5, a close to Gaussian emission profile has been obtained, it is shown in Fig. 6. With width of Gaussian fit $\sigma=2.50$ $\mathrm{mm}$ the ratio of initial beam radius to Sigma is: $r / \sigma=3.0$. Since the control electrode is permanently connected to the cathode, there is no need in constant component in a fitting function, which is just a Gaussian function now. 


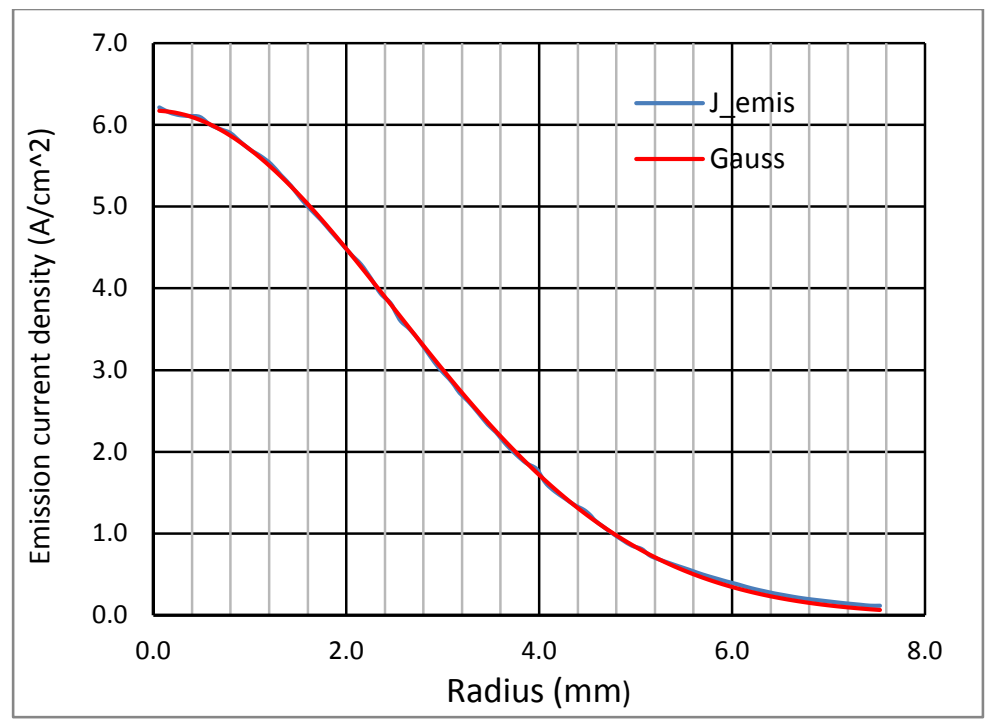

Fig. 6. Simulated radial profile of the emission current density of the second electron gun for anode voltage $U_{a n}=10.0 \mathrm{kV}$, control electrode voltage $U_{c e}=0.0 \mathrm{~V}$, electron current $\mathrm{I}_{\mathrm{el}}=2.49 \mathrm{~A}$. A fitting function has amplitude $\mathrm{A}=6.17 \mathrm{~A} / \mathrm{cm}^{2}$ and width $\sigma=2.50 \mathrm{~mm}$. The value of Error parameter is

$$
\mathrm{Er}=1.38 \mathrm{E}-4 \text {. }
$$

We found, that some of cathodes for the first gun had somewhat flattered tops, which resulted in a reduction of the emission current density in this area compare to the Gaussian distribution to a degree when a "well" was formed on the top of the emission radial profile.

Two cases of the cathode shape distortion have been simulated: a depression of the top by $0.05 \mathrm{~mm}$, which is smoothly distributed over radius of $1 \mathrm{~mm}$ and over radius of $3 \mathrm{~mm}$ (see Fig. $7 \mathrm{a}$ and $\mathrm{b}$ ).

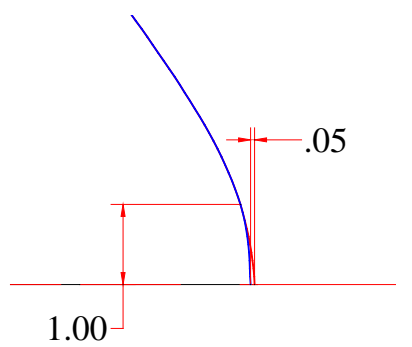

a

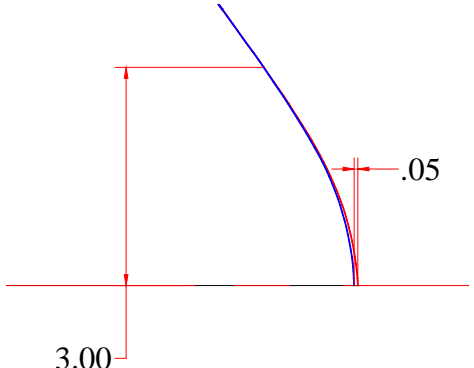

b

Fig. 7. Simulated geometries of "flattening" the top of the cathode by $0.05 \mathrm{~mm}$ on $\mathrm{r}=1 \mathrm{~mm}(\mathrm{a})$ and on $\mathrm{r}=3 \mathrm{~mm}$ (b). Red - original shape, which gives radial emission profile close to Gaussian, blue distorted shape.

Both cases has been simulated and the resulting emission profiles are presented in Fig. 8. 


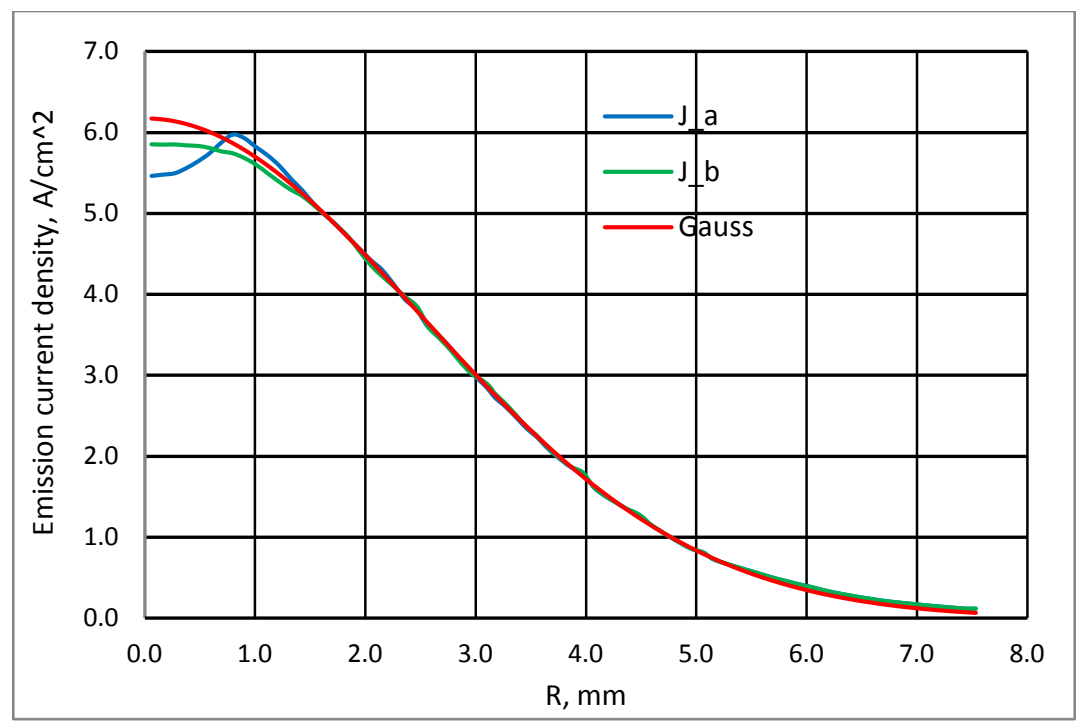

Fig. 8. Emission current density profiles for cathode surface distortions illustrated in Fig. 7 a (flattened over $1 \mathrm{~mm}$ ) and b (flattened over $3 \mathrm{~mm}$ ). The red curve is a Gaussian fit for not-distorted cathode.

One can see that distributing the depression of the cathode height over a larger radius helps preserving the Gaussian distribution to some extent.

We studied effect of gun geometry variations on a gun performance, including values of beam width $\sigma$, perveance and deviation from the Gaussian distribution, the Error parameter. To evaluate the effect of axial position of the control electrode with respect to the cathode on the performance of the electron gun we simulated the gun with different distances between the cathode and the control electrode. The position of the anode with respect to the cathode in this set of simulations remained the same. The result is presented in Fig. 9.

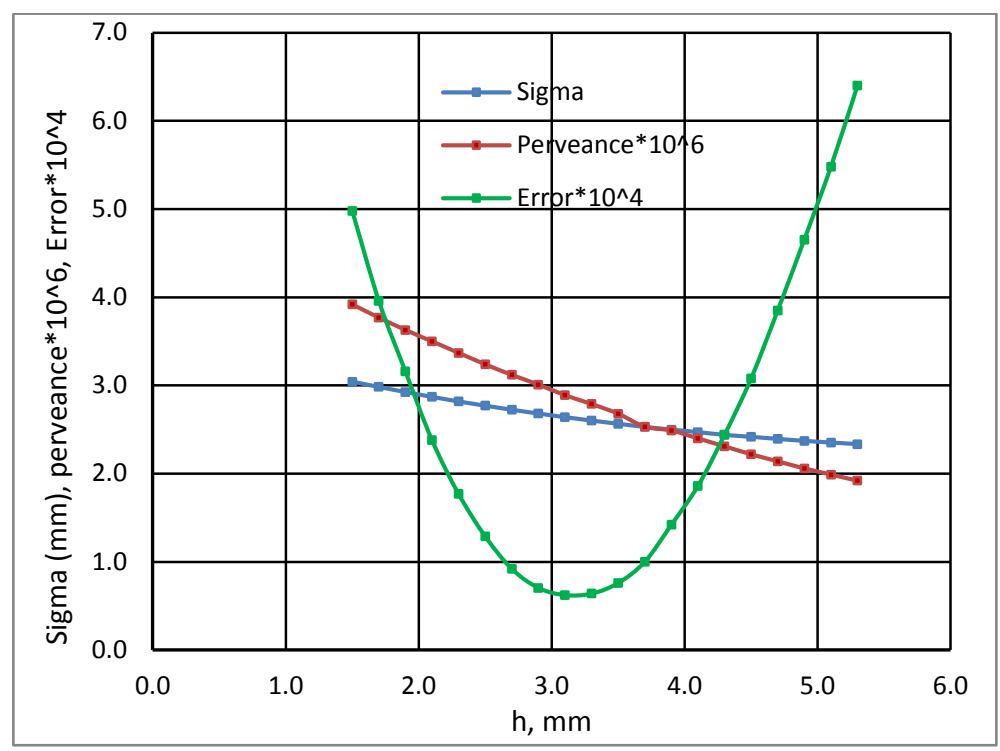

Fig. 9. Dependence of Sigma, gun perveance and Error parameter on the distance between the cathode and the control electrode. 
Curiously, the optimum distance between the cathode and the control electrode appeared to be 0.8 mm shorter, than it was in an initial geometry. At the optimum position the error function is 2 times smaller than it is for an initial geometry and the increase in value of Sigma from the original to optimum position is very small. The rise of Error parameter at larger and smaller cathode-control electrode distances is caused primarily by an increased emission on the periphery of the cathode compare to the Gaussian distribution. Perveance dependence on the cathode-control electrode distance is caused by shielding effect of the control electrode. But increasing the gun perveance by reducing the cathode-control electrode distance can be acceptable only within a limited range of this distance. It seems, deviations from the optimum distance by $\pm 0.5 \mathrm{~mm}$ can be acceptable, since the error parameters in this range is $\leq 1 \mathrm{E}-4$.

A sensitivity of the electron gun parameters to the inner diameter (ID) of the control electrode has been simulated and the result of these simulations is presented in Fig. 10. The cathode-control electrode distance remained fixed at the optimum distance of $3.1 \mathrm{~mm}$ and the cathode - anode distance was also fixed at initial value of $9.0 \mathrm{~mm}$.

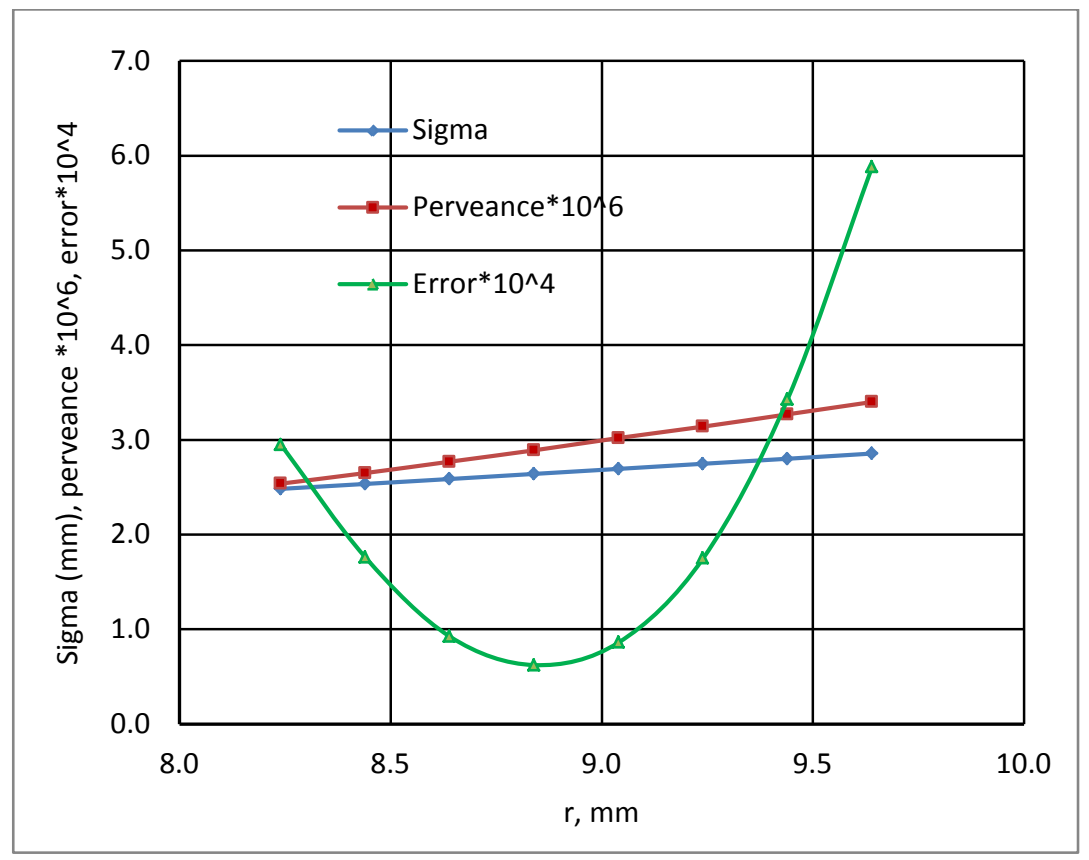

Fig. 10. Dependence of Sigma, gun perveance and Error parameter on the inner radius of the control electrode.

As expected, the minimum in error parameter distribution is at the original value of inner radius of the control electrode $(8.84 \mathrm{~mm})$. Deviations of ID by $\pm 0.5 \mathrm{~mm}$ can be tolerated since in this range the error parameter stays below $1 \mathrm{E}-4$.

A similar analysis has been done for a cathode-anode distance. Fig. 11 demonstrates gun performance parameters as a function of this distance. 


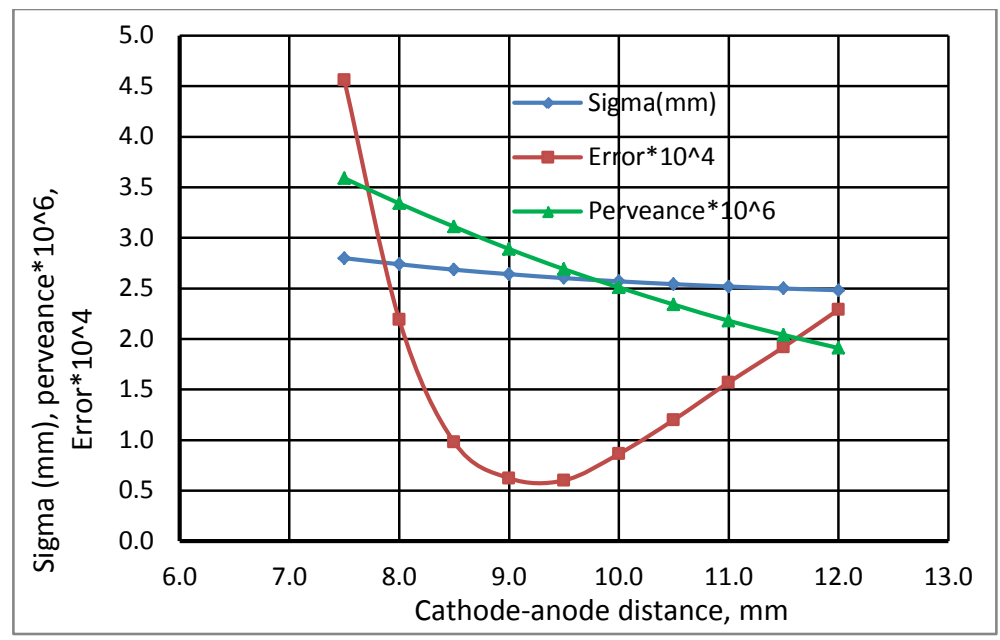

Fig. 11. Dependence of Sigma, perveance and error on a cathode - anode distance.

This plot demonstrates that deviation of the emission profile from Gaussian is smallest if the cathode-anode distance $\mathrm{CA}=9.0 \mathrm{~mm}$, where the error parameter has minimum. The error distribution is not symmetrical with respect to the optimum position and the error grows faster in the direction of smaller distances. The increase in perveance by reducing the cathode - anode distance (CA) can only be tolerated within a range of $8.5 \leq C A \leq 10.2$, which gives quite visible range of the gun perveance $\mathrm{P}: 3.11 \cdot 10^{-6} \leq \mathrm{P} \leq 2.4 \cdot 10^{-6}$.

\section{Electric field analysis.}

The simulated electric field in a cathode - anode gap is presented in Fig. 12:
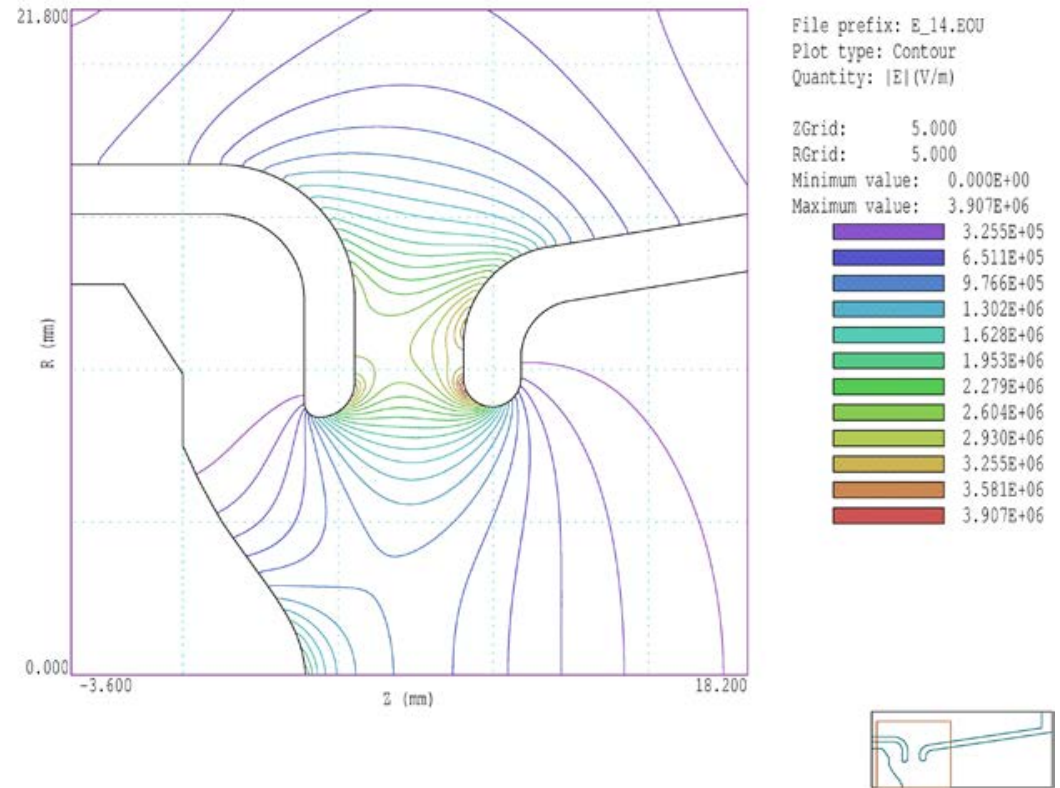

Fig. 11. Electric field distribution in a cathode - anode gap of the electron gun. $U_{\text {anode }}=10.0 \mathrm{kV}$.

At anode voltage $10 \mathrm{kV}$ the maximum electric field on the control electrode is $3.2 \mathrm{kV} / \mathrm{mm}$. The Kilpatrick safety factor is 6.3. 


\section{Conclusion.}

- Both versions of the electron gun for RHIC e-lens with cathode diameters $8.2 \mathrm{~mm}$ and 15.0 mm satisfy requirements to a current density distribution.

- The second electron gun has been optimized for the electrodes positioning and ID of the control electrode.

- Tolerances for the electrodes positions and the control electrode ID have been established.

- The second electron gun has much higher perveance than the first gun, and yet its anode voltage holdoff appears to be much better.

\section{References:}

[1] V. Kamerdzhiev, G. Kuznetsov, V. Shiltsev, N. Solyak, M. Tiunov, Electron beam generation in Tevatron electron lenses, 12th Advanced Accelerator Concepts Workshop, AIP Conference Proceedings, Volume 877, pp. 609-615 (2006).

[2] A. Pikin, W. Fischer, J. Alessi, M. Anerella, E. Beebe, D. Gassner, X. Gu, R. Gupta, J. Hock, R. Lambiase, Y. Luo, C. Montag, M. Okamura, Y. Tan, J. Tuozzolo, P. Thieberger, W. Zhang, Structure and Design of the Electron Lens for RHIC, Proceedings of 2011 Particle Accelerator Conference, New York, 2011, TPH100,

[3] http://www.fieldp.com/

[4] X. Gu, Z. Altinbas, M. Anerella, D. Bruno, M. Costanzo, W.C. Dawson, A.K. Drees, W. Fischer, B. M. Frak, D.M. Gassner, K. Hamdi, J. Hock, L.T. Hoff, A.K. Jain, J. Jamilkowski, R. Lambiase, Y. Luo, M. Mapes, A. Marone, C. Mi, R. Michnoff, T. Miller, M. Minty, C. Montag, S. Nemesure, W. Ng, D. Phillips, A.I. Pikin, S.R. Plate, P. J. Rosas, J. Sandberg, P. Sampson, L. Snydstrup, Commissioning RHIC’s electron lens, PAC 2013, Pasadena, TUOCA2. 